\title{
Esophageal cancer tumorspheres involve cancer stem-like populations with elevated aldehyde dehydrogenase enzymatic activity
}

\author{
GONG ZHANG $^{1 *}$, LEI MA $^{2 *}$, YOU-KE XIE $^{1 *}$, XIAO-BO MIAO $^{1}$ and CHUAN JIN ${ }^{2}$ \\ ${ }^{1}$ Cancer Research Institute of Southern Medical University, Guangzhou 510515; ${ }^{2}$ Internal Medical Department \\ of Guangzhou Medical University Cancer Institute and Hospital, Guangzhou 510182, P.R. China
}

Received February 29, 2012; Accepted June 1, 2012

DOI: $10.3892 / \mathrm{mmr} .2012 .939$

\begin{abstract}
Cancer stem cells (CSCs) form spheres in vitro in serum-free suspension culture. Sphere formation is particularly useful to enrich the potential CSC subpopulations as a functional approach. Few reports are currently available on tumorspheres in esophageal cancer (EC). The present study focused on evaluating the cancer stem-like properties and analyzing the difference between spheroid and adherent cells of the Eca109 human EC cell line. Immunofluorescence and immunoblotting analysis revealed that EC tumorspheres expressed the stem cell markers Nanog and Oct4 more highly, but showed a decreased expression of the differentiation marker CK5/6. The spheroids were chemoresistant to cisplatin compared to the adherent cells (32.5 vs. $135.8 \mu \mathrm{M}$ in $\mathrm{IC}_{50}$ ). Side population cells increased in tumorspheres compared to adherent cells (0.7 vs. 5.6\%). A marked upregulation of drug-resistant genes (ABCG2 and MDR1) was observed in sphere-forming cells. We compared the profiles of adherent and spheroid cells by microarrays and obtained one representative differentially expressed gene, aldehyde dehydrogenase (ALDH). We also verified that the cancer stem-like cells of EC contained a high ALDH enzymatic activity. ALDH-positive cells were enriched by 11 - to 12-fold in spheroids, compared to adherent cells (2.5 vs. $28.6 \%$ ). Immunofluorescence and immunoblotting analysis also revealed a higher expression of ALDH in EC tumorspheres. In conclusion, our study verified that sphere-forming culturing can be utilized to demonstrate the putative esophageal CSCs, and identified a potential esophageal CSC surface marker, ALDH.
\end{abstract}

Correspondence to: Dr Chuan Jin, Internal Medical Department of Guangzhou Medical University Cancer Institute and Hospital, Luhu Street, Guangzhou 510182, P.R. China

E-mail: jinchuan361@163.com

*Contributed equally

Key words: esophageal cancer, cancer stem cells, tumorsphere, microarray, aldehyde dehydrogenase

\section{Introduction}

Esophageal cancer (EC) is a highly malignant neoplasm. The 5 -year survival rate of patients is only $10 \%$ (1). Advanced EC is one of the most refractory cancers and is associated with poor outcome. Conventional chemotherapy and radiotherapy are widely used for EC. However, more than $40 \%$ of EC cases eventually result in recurrence and patients succumb to chemotherapy- and radiotherapy-resistant disease (2). Mounting evidence suggests that small populations of cells within tumors, known as cancer stem cells (CSCs), contribute to tumor maintenance and progression and are intrinsically resistant to therapies (3). CSCs have the ability to recreate the full phenotypic heterogeneity of the parent tumor (4). These cells express distinct surface markers allowing for reproducible and differential purification. Several stem cell markers, such as Nanog and Oct4, have been used successfully to identify CSCs in normal and tumor tissue (5). In addition, side population (SP) cells found in various types of cancer have been reported to exhibit CSC characteristics (6).

The anchorage-independent tumorsphere culture of stem cells was instrumental in the study of adult CSCs (7-9). Sphere formation is particularly useful to enrich the potential CSC subpopulations as a functional approach $(10,11)$. CSCs form spheres in vitro in serum-free suspension culture. In the suspension culture, tumorsphere-forming cells failed to express cytokeratins (CK), but were found to express stem cell markers (12). Thus, the suspension culture system is thought to maintain CSCs in their undifferentiated state, facilitating their enrichment.

However, few reports are currently available regarding tumorspheres in EC. Therefore, the aim of the present study was to enrich and identify EC cell subsets with CSC properties. The tumorsphere of $\mathrm{EC}$ is considered to be a valuable model for the further study of both CSCs and chemoresistance. To select esophageal CSC markers, we performed comparative global gene expression analyses between adherent and spheroid cells. We compared profiles of adherent and spheroid cells and obtained one representative differentially expressed gene, aldehyde dehydrogenase (ALDH). We also verified that the cancer stem-like cells of EC contained a high ALDH enzymatic activity. 


\section{Materials and methods}

Cells and culture conditions. The Eca109 human esophageal cancer cell line was purchased from the Shanghai Cell Biology Institute of the Chinese Academy of Sciences, China. The cells were cultured in DMEM medium (Hyclone, Logan, UT, USA) supplemented with $10 \%$ fetal bovine serum (FBS; Hyclone) and $100 \mathrm{U} / \mathrm{ml}$ penicillin/streptomycin (Gibco, Langley, OK, USA). Cultures were maintained in a humidified incubator at $37^{\circ} \mathrm{C}$ in $5 \% \mathrm{CO}_{2}$ air atmosphere.

Tumorsphere culture and differentiation. Cells $(1,000$ cells $/ \mathrm{ml})$ were cultured in suspension in serum-free Ham's F-12 medium (Gibco), supplemented with B27 (1:50; Gibco), $20 \mathrm{ng} / \mathrm{ml}$ EGF (Invitrogen, Grand Island, NY, USA) and $20 \mathrm{ng} / \mathrm{ml} \mathrm{FGF}$ (Invitrogen). To propagate spheres in vitro, spheres were collected by gentle centrifugation, dissociated to single cells and then cultured to generate tumorspheres of the next generation. To guide the differentiation of spheres in vitro, spheroids were cultured in DMEM supplemented with $10 \%$ FBS without growth factors.

Immunofluorescent staining. For immunofluorescent staining, adherent or semi-differentiated spheroid cells were grown on the surface of cover slides. Spheroid staining was performed in 96-well microplates. The cells were fixed with $4 \%$ paraformaldehyde. Following rehydration in PBS, cells were incubated with respective primary antibodies at $37^{\circ} \mathrm{C}$ for $45 \mathrm{~min}$. Mouse anti-Nanog, Oct4, CK5/6 and ALDH1 (Santa Cruz Biotechnology, Santa Cruz, CA, USA) were used as primary antibodies. Slides or spheroids were then washed with PBS for $15 \mathrm{~min}$ and secondary antibodies were incubated at $37^{\circ} \mathrm{C}$ for $45 \mathrm{~min}$. Alexa594-conjugated goat anti-mouse IgG (against anti-Nanog, Oct4 and CK5/6; Invitrogen) or FITC-conjugated goat anti-mouse IgG (against anti-ALDH1; Invitrogen) were used as secondary antibodies. The nuclei were stained with DAPI. Sections were examined with confocal microscopy (Olympus-FV1000, Japan).

Immunoblotting. Total protein was extracted from spheroid or adherent Eca109 cells using cell lysis buffer. Proteins were run in $10 \%$ SDS-PAGE and transferred on a PVDF sheet. The blots were incubated for $1-2 \mathrm{~h}$ in blocking solution $(5 \%$ skimmed milk in Tris-buffer), and then for $1 \mathrm{~h}$ using the following primary antibodies: mouse anti-Nanog, Oct4, CK5/6, ABCG2, MDR1,ALDH1 and GAPDH (Santa Cruz). The sheet was then incubated for $1 \mathrm{~h}$ with HRP-conjugated secondary antibodies (Invitrogen) against mouse immunoglobulins. The bands were visualized using the ECL-Plus detection system (Bio-Rad, Hercules, CA, USA).

Drug sensitivity assay to antitumor drug. Cells obtained from adherent or spheroid Eca109 cells were seeded in 96-well microplates at a density of 3,000 cells/well. The cells were treated with increasing concentrations of cisplatin (Sigma-Aldrich, St. Louis, MO, USA) as indicated by the manufacturer's instructions. MTT assay was performed to determine the cell viability following exposure to cisplatin for $72 \mathrm{~h}$. The number of living cells was directly proportional to the absorbance at $490 \mathrm{~nm}$.
Hoechst staining and SP cell assay. Cells obtained fromadherent or spheroid Eca109 cells were suspended in DMEM/2\% FBS at $1 \times 10^{6}$ cells $/ \mathrm{ml}$ and stained with Hoechst-33342 dye $\left(5 \mu \mathrm{g} / \mathrm{ml}\right.$; Sigma-Aldrich) for $90 \mathrm{~min}$ at $37^{\circ} \mathrm{C}$. Following this incubation, cells were washed with ice-cold PBS and stained with propidium iodide $(1 \mu \mathrm{g} / \mathrm{ml}$; Sigma-Aldrich) to label and exclude dead cells. The cells were maintained at $4^{\circ} \mathrm{C}$ for the flow cytometric analysis and for sorting of the SP fraction using a FACSAria flow cytometer (BD Biosciences, San Jose, CA, USA).

RNA isolation and microarray analysis. Eca109 spheroids were filtered by a cell strainer ( $40 \mu \mathrm{m}$; BD Biosciences). Spheroids with a diameter of $>40 \mu \mathrm{m}$ were selected. Total RNA was extracted separately from adherent and spheroid Eca109 cells using TRIzol reagent (Invitrogen), according to the manufacturer's instructions. RNA was subjected to GeneChip_ expression array analysis with two-cycle target labeling (implemented by CapitalBio Corp., Beijing, China). Briefly, cDNA was synthesized from total RNA using T7-Oligo (dT) primers, and biotinylated cRNA was synthesized using cDNA. Labeled cRNA ( $2 \mu \mathrm{g})$ was hybridized to the 22K Human Genome Array. The array image was scanned and analyzed using LuxScan 10KA.

Aldefluor assay by FACS. The ALDEFLUOR kit (StemCell Technologies, Durham, NC, USA) was used to analyze the population with a high ALDH enzymatic activity. Cells obtained from adherent or spheroid Eca109 cells were suspended in ALDEFLUOR assay buffer containing ALDH substrate and incubated during $40 \mathrm{~min}$ at $37^{\circ} \mathrm{C}$. As a negative control, for each sample of cells an aliquot was treated with $50 \mathrm{mmol} / \mathrm{l}$ diethylaminobenzaldehyde (DEAB), a specific ALDH inhibitor. FACS was performed using a FACSAria flow cytometer (BD Biosciences).

Statistical analyses. Data were analyzed using statistics soft SPSS 13.0 and were shown as the means \pm SD. P $<0.05$ were considered statistically significant.

\section{Results}

Esophageal cancer tumorsphere contains cells with cancer stem-like properties. Ponti et al first reported that breast CSC properties could be propagated in vitro as non-adherent mammospheres under serum-free culture conditions (13). In the present study, we attempted to enrich the CSC population from the Eca109 EC cell line. To observe the differentiation of the tumorspheres, spheres were cultivated in serum-driven culture. After $48 \mathrm{~h}$ of culture, floating undifferentiated cells attached to the plastic, gradually migrating from tumorspheres and differentiating into adherent cells (Fig. 1A). We detected two typical CSC markers, Nanog and Oct4, that were spheroid-cultured under differentiation conditions by immunofluorescence. In addition, the expression of the marker which indicates EC surface epithelium, CK5/6, was also observed. As shown in Fig. 1B, Nanog and Oct4 were expressed in the center of the semi-differentiated spheroids. However, a markedly decreased expression was observed at the edge of the semi-differentiated spheroids. Inversely, CK5/6 expression was almost absent in 
A
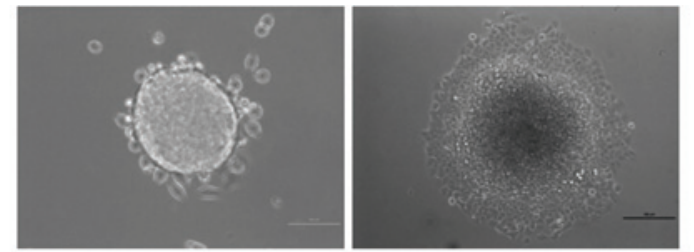

B

DAPI
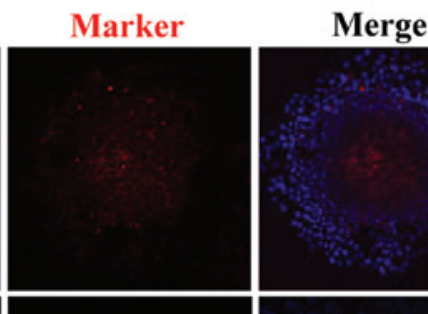

Nanog

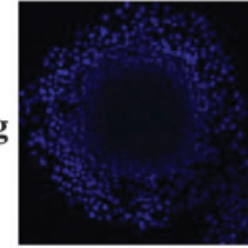

Oct4
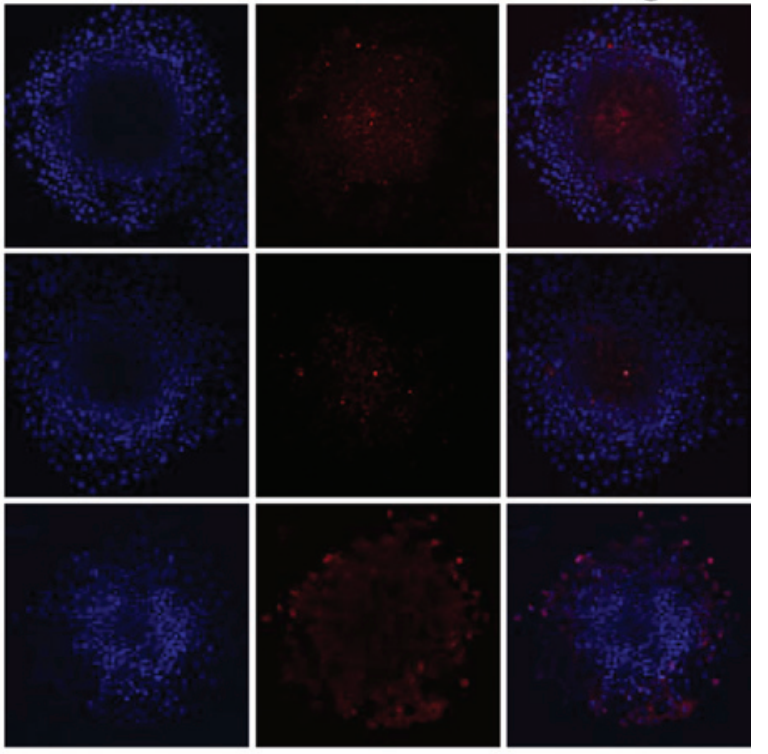

CK5/6
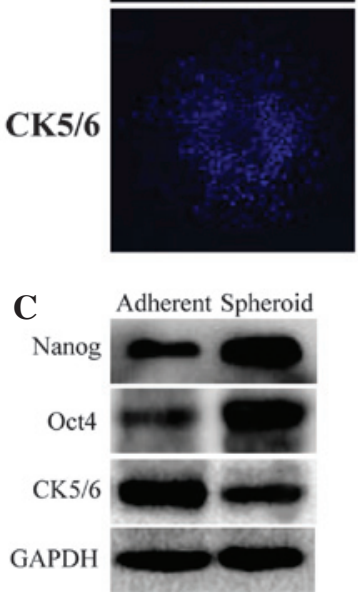

Figure 1. (A) Microscopical analysis of Eca109 EC tumorsphere cultivated in serum-free (left panel) and differentiation conditions for $48 \mathrm{~h}$ (right panel). Scale bars, $100 \mu \mathrm{m}$. (B) Spheroids of Eca109 cells were cultured under serum-driven cultures for $48 \mathrm{~h}$. The indicated antibodies were analyzed by immunofluorescence using a confocal laser scanning microscope. (C) Expression levels of Nanog, Oct 4 and CK5/6 in the adherent and spheroid Eca109 cells examined by immunoblotting are shown.

the center of the semi-differentiated spheroids, but was markedly expressed at the edge of the semi-differentiated spheroids.

Cancer stem-like properties were confirmed at the protein level in EC spheroids by immunoblotting. As expected, cancer cells cultured in the serum-free medium caused a CSC marker shift in the cells, including a marked upregulation of the CSC markers Nanog and Oct4, and the downregulation of the epithelium marker CK5/6 (Fig. 1C). The results indicated that EC tumorspheres demonstrated an increased expression of stem cell markers.

EC tumorspheres exhibit an increased expression of $A B C$-transporter and resistance to chemotherapeutic drugs. To examine whether EC tumorspheres possess a hypothesized chemoresistant phenotype of the CSCs, we assessed the sensitivity of the sphere-forming cells and the differentiated cells to drugs commonly used in chemotherapy. The EC tumor cells from the spheroids exhibited an increased $\mathrm{IC}_{50}$ (half maximal
A

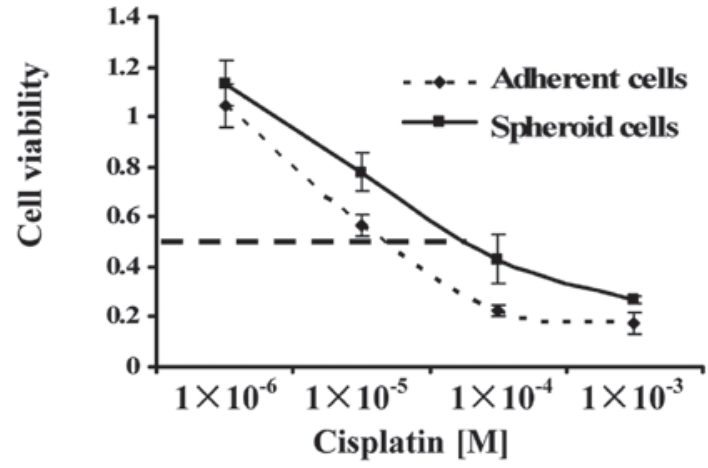

B
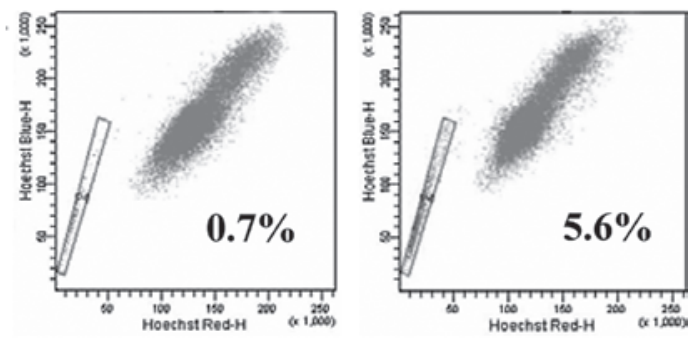

C

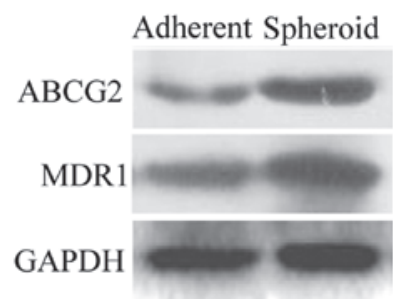

Figure 2. (A) Dose-response curves of cells obtained from adherent and spheroid Eca109 cells following $72 \mathrm{~h}$ of treatment with cisplatin. Bars are the standard error $(n=3)$. (B) Comparative FACS analysis of SP cells of adherent (left panel, 0.7\%) and spheroid (right panel, 5.6\%) Eca109 cells. (C) Expression levels of ABCG2 and MDR1 in the adherent and spheroid Eca109 cells examined by immunoblotting.

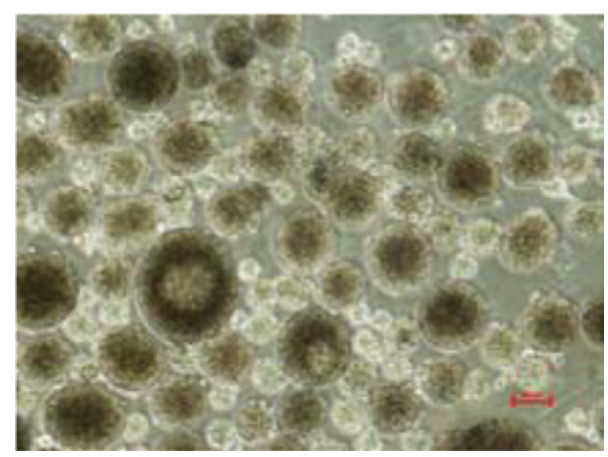

Figure 3. Image of spheroids with a diameter of $>40 \mu \mathrm{m}$ which were selected to perform the microarray analysis. Scale bars, $100 \mu \mathrm{m}$.

inhibitory concentration) value (4- to 5 -fold; 32.5 vs. $135.8 \mu \mathrm{M}$ ) to cisplatin compared to the control adherent cells (Fig. 2A). Tumor cells resistant to chemotherapy occur in part due to the overexpression of ATP-binding cassette multidrug-resistance gene1 (MDR1) (14) and ATP-binding cassette sub-family G member 2 (ABCG2) (15). This property correlates with the ability to expel dyes, defined as a flow cytometry SP (6). SP cells have also been reported to exhibit CSC characteristics (16). In our study, EC cells cultured in suspension cultures were found to contain an 8-fold increase in the proportion of SP 
Table I. Differentially expressed genes (sphere vs. adherent).

\begin{tabular}{|c|c|c|c|}
\hline ID & Gene symbol & Ratio & Gene description \\
\hline 12964 & EREG & 38.0760 & Epiregulin precursor \\
\hline 5324 & $\mathrm{~S} 100 \mathrm{~A} 2$ & 36.6280 & S100 calcium-binding protein $\mathrm{A} 2$ \\
\hline 13689 & IL1B & 25.3600 & Interleukin-1 $\beta$ precursor \\
\hline 9469 & DUSP6 & 19.1750 & Dual specificity protein phosphatase 6 \\
\hline 10355 & $\mathrm{TNC}$ & 17.8630 & Tenascin precursor \\
\hline 13167 & IGFBP7 & 16.8850 & Insulin-like growth factor binding protein 7 precursor \\
\hline 4767 & SAT & 11.5940 & Diamine acetyltransferase 1 \\
\hline 7765 & CLECSF2 & 11.5100 & C-type lectin superfamily member 2 \\
\hline 20212 & EGR1 & 10.4540 & Early growth response protein 1 \\
\hline 4340 & FOS & 9.6953 & Proto-oncogene protein c-fos \\
\hline 5981 & KLK11 & 8.8023 & Kallikrein 11 precursor \\
\hline 16753 & FHL1 & 8.3500 & Skeletal muscle LIM-protein 1 \\
\hline 7978 & MMP1 & 8.3340 & Interstitial collagenase precursor \\
\hline 7871 & HAS3 & 8.1108 & Hyaluronan synthase 3 \\
\hline 15869 & TNFAIP3 & 7.3406 & Tumor necrosis factor, $\alpha$-induced protein 3 \\
\hline 12711 & COL17A1 & 7.2343 & Collagen $\alpha 1$ (XVII) chain \\
\hline 1415 & IL1A & 7.2178 & Interleukin-1 $\alpha$ precursor \\
\hline 14111 & SERPINB7 & 7.0842 & Megsin \\
\hline 8895 & GBP2 & 6.6438 & Interferon-induced guanylate-binding protein 2 \\
\hline 958 & LTB & 6.5935 & Lymphotoxin- $\beta$ \\
\hline 5021 & ALDH1A1 & 6.5078 & Aldehyde dehydrogenase 1 family, member A1 \\
\hline 1813 & TIMP1 & 6.5020 & Metalloproteinase inhibitor 1 precursor \\
\hline 6918 & SERPINB2 & 6.4078 & Plasminogen activator inhibitor-2 precursor \\
\hline 7661 & S100A4 & 6.3883 & S100 calcium-binding protein A4 \\
\hline 6163 & GPR87 & 6.3117 & Probable $\mathrm{G}$ protein-coupled receptor GPR8 \\
\hline 6936 & ALDH1A3 & 6.0678 & Aldehyde dehydrogenase 6 \\
\hline 2523 & FST & 6.0256 & Follistatin precursor \\
\hline 5966 & LAMC2 & 5.9743 & Laminin $\gamma-2$ chain precursor \\
\hline 6517 & $\mathrm{BF}$ & 5.9716 & Complement factor B precursor \\
\hline 1393 & FGFBP1 & 5.8656 & Fibroblast growth factor binding protein 1 \\
\hline 5322 & TSN1 & 5.6896 & Tetraspanin 1 \\
\hline 3284 & PLAG1 & 5.6665 & Pleiomorphic adenoma gene 1 \\
\hline 3883 & PHCA & 5.4134 & Alkaline phytoceramidase \\
\hline 6664 & C10orf116 & 5.4024 & Adipose most abundant gene transcript 2 \\
\hline 1512 & CXCL10 & 5.2340 & Small inducible cytokine B10 precursor \\
\hline 3028 & SNX8 & 5.1606 & Sorting nexin 8 \\
\hline 968 & ALDH3A1 & 5.1533 & Aldehyde dehydrogenase, dimeric NADP-preferring \\
\hline 1336 & SEMA3A & 5.0699 & Semaphorin 3A precursor \\
\hline 17731 & MA17 & 5.0025 & $17 \mathrm{kDa}$ membrane associated protein \\
\hline
\end{tabular}

Bold indicates $>5$-fold difference in the expression of the gene involved.

cells compared to the adherent controls (0.7 vs. 5.6\%; Fig. 2B). ABCG2 and MDR1 were also confirmed at the protein level by immunoblotting. The result indicated that ABCG2 and MDR1 were substantially increased in tumorspheres compared to the adherent cells (Fig. 2C).

Gene expression profile analysis of EC spheroids based on microarray data. To clarify differential gene expression profiles between tumorsphere and the adherent cells of EC, microarray analysis was performed. A previous study has verified that the more serial passages in the spheroids, the more CSCs in spheroids (12). To ensure the reliability of microarray results, we achieved the 20th passage of EC spheroids. The spheroids were filtered by a cell strainer. Spheroids with a diameter of $>40 \mu \mathrm{m}$ were selected to perform the microarray analysis (Fig. 3). The mRNA expression profiles of the spheroid and adherent Eca109 cells were analyzed by human cDNA microarray. Among the 21,522 probes examined, 
376 genes were upregulated (ratio >2.0) in the spheroid cells compared to the adherent cells, whereas 325 genes were downregulated in the spheroid cells. The upregulated genes were then assigned to a functional class using a gene ontology annotation tool by the Database for Annotation, Visualization and Integrated Discovery (Bioresource for array genes; http://david.abcc.ncifcrf.gov/). Based on their functions, the majority of these genes were classified into 'polymorphism', 'extracellular matrix', 'phosphoprotein', 'cell adhesion' and 'cell secretion' groups. In addition, we found 39 genes that showed a $>5$-fold upregulation in the spheroid cells compared to the adherent cells (Table I). Among these genes, three upregulated genes of the ALDH family exhibited a $>5$-fold difference in expression, including ALDH1A1, ALDH1A3 and ALDH3A1 (Table I, bold).

EC spheroids contain high ALDH enzymatic activity. In the different profiles, we found that three ALDH family-related genes were significantly upregulated in the tumorsphere. ALDH, which detoxifies intracellular aldehydes through oxidation, may have a role in the differentiation of stem cells through the oxidation of retinoic acid. (17) ALDH expression has been suggested as a potential functional marker for CSCs (18). To confirm this finding, we utilized the ALDEFLUOR assay to assess the size of the population with ALDH enzymatic activity in the Eca109 EC cell line. ALDEFLUORpositive cells were enriched by 11 - to 12 -fold in spheroids, compared to the adherent cells (2.5 vs. $28.6 \%$; Fig. $4 \mathrm{~A})$. The ALDH increase in the tumorsphere was further confirmed at the protein level by immunofluorescence and immunoblotting. Immunofluorescence indicated that ALDH1 expression was observed in the tumorspheres, but was markedly decreased in the adherent cells (Fig. 4B). Immunoblotting showed a similar result; ALDH1 was found to be upregulated in tumorspheres compared to the control adherent cells (Fig. 4C). These results suggest that ALDH1-positive cells represent the stem/ progenitor population of EC.

\section{Discussion}

Current therapies for EC eliminate most cells within a tumor. However, advanced EC still progresses to incurable, androgenindependent metastatic disease (19). According to the CSC hypothesis, current therapies fail to prevent cancer relapse and metastasis, since the small population of tumor stem cells is not susceptible to therapy (3). The tumorsphere, SP cells and drug-resistant cells have cancer stem-like properties. The SP technique is widely used to identify stem-like cells in cancer cells (20). SP cells derived from primary EC cells were more resistant to chemotherapeutic reagents and formed more colonies in vitro than non-SP cells; xenograft experiments revealed that SP cells were more tumorigenic in vivo (21). Drug resistance-related gene ABCG2 expression is an independent unfavorable prognostic factor in esophageal squamous cell carcinoma (22).

Previous studies have reported the application of sphere culture to isolate, enrich, maintain or expand potential CSC subpopulations from various types of cancer (23-27). It is generally agreed that, as with all stem cells, the tumorsphereforming cells are capable of proliferation and self-renewal and
$\mathbf{A}$
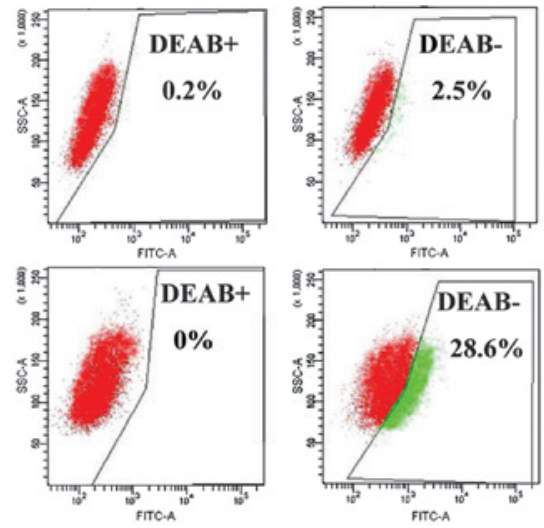

B

DAPI
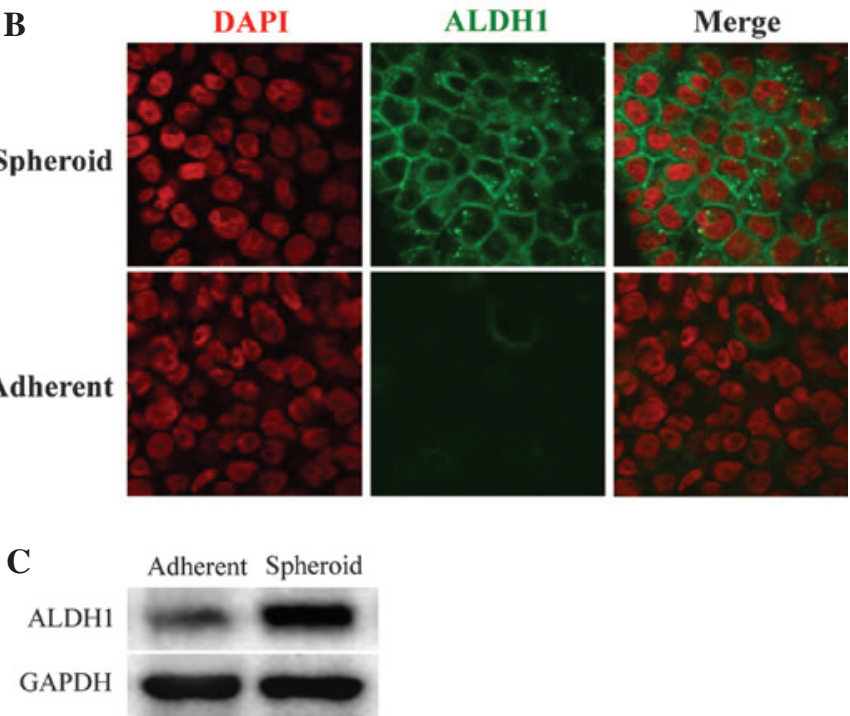

Figure 4. (A) Comparative FACS analysis of ALDH-positive cells of adherent (upper, 2.5\%) and spheroid (bottom, 28.6\%) Eca109 cells. (B) ALDH1 expression of spheroid and adherent Eca109 cells was analyzed by immunofluorescence using a confocal laser scanning microscope. (C) Expression levels of ALDH1 in the spheroid and adherent Eca109 cells examined by immunoblotting.

possess higher tumorigenicity. To the best of our knowledge, few reports are available on the propagation of esophageal CSCs using sphere culture. In the present study, we provide a systematic investigation of sphere-propagating cells that are derived from the Eca109 EC cell line.

The hypothesis that our tumorspheres exhibitied stem-like properties was based on the following observations: i) Nanog and Oct 4 were expressed in the undifferentiated spheroid cells, but the expression of CK5/6 was markedly decreased in undifferentiated spheroid cells; ii) spheroid cells contain more Nanog and Oct4 protein, but less CK5/6 protein than adherent cells; iii) tumorspheres exhibited an increased resistance to cisplatin; iv) spheroid cells had an increased prevalence of SP cells and v) the ABC-transporter protein was enriched in spheroid cells compared to adherent cells. Therefore, we suggest that the non-adherent tumorspheres cultured in serum-free conditions possess esophageal CSC properties. Thus, suspension culture may effectively be used to enrich esophageal CSCs.

To understand the mechanisms underlying the difference in spheroid and adherent cells in the Eca109 cell line, we performed gene chip analysis and found that three genes from the ALDH family were highly expressed in esophageal cancer 
stem-like cells. This observation was further confirmed by immunoblotting. ALDHs are a superfamily of 17 intracellular enzymes that protect cells from the cytotoxic effects of peroxic aldehydes (28). Increased ALDH activity has also been found in stem cell populations in various types of cancer (18). ALDH activity may therefore provide a marker for normal and malignant stem as well as progenitor cells. Our study indicated that a high ALDH enzymatic activity is a function of EC tumorsphere. ALDH1 expression has been confirmed to associate with lymph node metastasis and poor survival in EC (29). Thus, we believe that ALDH is a putative CSC marker of EC.

In conclusion, our study outlines a condition for long-term culture of EC stem-like cells. This system is likely to be beneficial for the investigation of unique properties of EC stem-like cells in terms of their biology and their specific cell surface marker expression that distinguishes them from common EC cells. Regarding specific surface markers that are associated with stem-like cells, our current understanding is that ALDH is a potential esophageal CSC surface marker. Nevertheless, esophageal CSC cell surface markers remain to be identified. EC tumorsphere and our tumorsphere microarray analysis data provide a unique opportunity to find and identify such markers.

\section{Acknowledgements}

This study was supported by a Grant from the Technology Program of the Science and Technology Department of Guangdong Province (no. 2011B080701021).

\section{References}

1. Ekman S, Dreilich M, Lennartsson J, Wallner B, Brattström D, Sundbom $\mathrm{M}$ and Bergqvist M: Esophageal cancer: current and emerging therapy modalities. Expert Rev Anticancer Ther 8: 1433-1448, 2008.

2. Aminian A, Panahi N, Mirsharifi R, Karimian F, Meysamie A, Khorgami Z and Alibakhshi A: Predictors and outcome of cervical anastomotic leakage after esophageal cancer surgery. J Cancer Res Ther 7: 448-453, 2011.

3. Dalerba P and Clarke MF: Cancer stem cells and tumor metastasis: first steps into uncharted territory. Cell Stem Cell 1: 241-242, 2007.

4. Dalerba P, Cho RW and Clarke MF: Cancer stem cells models and concepts. Annu Rev Med 58: 267-284, 2007.

5. Mathieu J,Zhang Z,Zhou W, et al: HIF induces human embryonic stem cell markers in cancer cells. Cancer Res 71: 4640-4652, 2011.

6. Greve B, Kelsch R, Spaniol K, Eich HT and Götte M: Flow cytometry in cancer stem cell analysis and separation Cytometry A 81: 284-293, 2012.

7. Dontu G, Abdallah WM, Foley JM, Jackson KW, Clarke MF, Kawamura MJ and Wicha M: In vitro propagation and transcriptional profiling of human mammary stem/progenitor cells. Genes Dev 17: 1253-1270, 2003.

8. Bez A, Corsini E, Curti D, Biggiogera M, Colombo A, Nicosia RF, Pagano SF and Parati EA: Neurosphere and neurosphere-forming cells: morphological and ultrastructural characterization. Brain Res 993: 18-29, 2003.
9. Shi X, Gipp J and Bushman W: Anchorage-independent culture maintains prostate stem cells. Dev Biol 312: 396-406, 2007.

10. Zhang S, Balch C, Chan MW, et al: Identification and characterization of ovarian cancer-initiating cells from primary human tumors. Cancer Res 68: 4311-4320, 2008.

11. Fujii H, Honoki K, Tsujiuchi T, Kido A, Yoshitani K and Takakura Y: Sphereforming stem-like cell populations with drug resistance in human sarcoma cell lines. Int J Oncol 34: 1381-1386, 2009.

12. Ricci-Vitiani L, Lombardi DG, Pilozzi E, Biffoni M, Todaro M, Peschle $\mathrm{C}$ and De Maria R: Identification and expansion of human colon-cancer-initiating cells. Nature 445: 111-115, 2007.

13. Ponti D, Costa A, Zaffaroni N, et al: Isolation and in vitro propagation of tumorigenic breast cancer cells with stem/progenitor cell properties. Cancer Res 65: 5506-5511, 2005.

14. Podolski-Renić A, Andelković T, Banković J, Tanić N, Ruždijić S and Pešić M: The role of paclitaxel in the development and treatment of multidrug resistant cancer cell lines. Biomed Pharmacother 65: 345-353, 2011.

15. Ding XW, Wu JH and Jiang CP: ABCG2: a potential marker of stem cells and novel target in stem cell and cancer therapy. Life Sci 86: 631-637, 2010.

16. Tabor MH, Clay MR, Owen JH, Bradford CR, Carey TE, Wolf GT and Prince ME: Head and neck cancer stem cells: the side population. Laryngoscope 121: 527-533, 2011.

17. Stagos D, Chen Y, Brocker C, et al: Aldehyde dehydrogenase 1B1: molecular cloning and characterization of a novel mitochondrial acetaldehyde-metabolizing enzyme. Drug Metab Dispos 38: 1679-1687, 2010.

18. Ma I and Allan AL: The role of human aldehyde dehydrogenase in normal and cancer stem cells. Stem Cell Rev 7: 292-306, 2011.

19. Kunisaki C, Makino H, Kimura J, et al: Therapeutic strategy for esophageal cancer based on solitary lymph node metastasis. Hepatogastroenterology 58: 1561-1565, 2011.

20. Wu C and Alman BA: Side population cells in human cancers. Cancer Lett 268: 1-9, 2008.

21. Li H, Gao Q, Guo L and Lu SH: The PTEN/PI3K/Akt pathway regulates stem-like cells in primary esophageal carcinoma cells. Cancer Biol Ther 11: 950-958, 2011.

22. Tsunoda $\mathrm{S}$, Okumura $\mathrm{T}$, Ito $\mathrm{T}$, et al: $\mathrm{ABCG} 2$ expression is an independent unfavorable prognostic factor in esophageal squamous cell carcinoma. Oncology 71: 251-258, 2006.

23. Hirschhaeuser F, Menne H, Dittfeld C, West J, Mueller-Klieser W and Kunz-Schughart LA: Multicellular tumor spheroids: an underestimated tool is catching up again. J Biotechnol 148: 3-15, 2010.

24. Singh SK, Clarke ID, Terasaki M, Bonn VE, Hawkins C, Squire J and Dirks P: Identification of a cancer stem cell in human brain tumors. Cancer Res 63: 5821-5828, 2003.

25. Gibbs CP, Kukekov VG, Reith JD, et al: Stem-like cells in bone sarcomas: implications for tumorigenesis. Neoplasia 7: 967-976, 2005.

26. Fang D, Nguyen TK, Leishear K, et al: A tumorigenic subpopulation with stem cell properties in melanomas. Cancer Res 65: 9328-9337, 2005.

27. Gou S, Liu T, Wang C, Yin T, Li K, Yang M and Zhou J: Establishment of clonal colony-forming assay for propagation of pancreatic cancer cells with stem cell properties. Pancreas 34: 429-435, 2007.

28. Ma S, Chan KW, Lee TK, Tang KH, Wo JY, Zheng BJ and Guan XY: Aldehyde dehydrogenase discriminates the CD133 liver cancer stem cell populations. Mol Cancer Res 6: 1146-1153, 2008.

29. Wang Y, Zhe H, Gao P, Zhang N, Li G and Qin J: Cancer stem cell marker ALDH1 expression is associated with lymph node metastasis and poor survival in esophageal squamous cell carcinoma: a study from high incidence area of northern China. Dis Esophagus: Nov, 2011 (E-pub ahead of print). 